\title{
An Equivalent Circuit Model for Nested Split-Ring Resonators
}

\author{
Burak Ozbey, Member, IEEE, Ayhan Altintas, Senior Member, IEEE, Hilmi Volkan Demir, Senior Member, IEEE, \\ and Vakur B. Ertürk, Member, IEEE
}

\begin{abstract}
In this paper, an equivalent circuit model for nested split-ring resonators (NSRRs) is proposed. NSRRs are an emerging class of split ring resonators, preferred in a range of areas from sensing in biomedical or civil engineering applications to antenna design, due to their more compact size and enhanced sensitivity/resolution characteristics over the conventional SRRs. In the proposed model, the NSRR structure is treated as a combination of basic elements, i.e., strips and gaps, and the electromagnetic characteristics of the whole geometry are expressed in terms of capacitances and inductances of each of these elements. The outputs of the model are compared with those obtained via full-wave simulations using the package programs as well as measurements. The variation of NSRR resonance frequency $\left(f_{\text {res }}\right)$ with all important design parameters is also compared with full-wave simulations. In all comparisons, the results demonstrate agreement, showing that the proposed model can correctly explain the electromagnetics of the NSRR structure and that it provides an intuitive way for a better and easier analysis and a preliminary design of normally complex structures.
\end{abstract}

Index Terms-Equivalent circuit model, nested split-ring resonator (NSRR), split-ring resonator (SRR), strain/displacement sensor, wireless passive sensor.

\section{INTRODUCTION}

$\mathbf{S}$ PLIT-RING resonators (SRRs) have traditionally been the building blocks of metamaterials or metamaterial-inspired structures since they were first proposed in [1]. Since then, SRRs have been exploited in a wide range of applications including transmission lines [2], antennas [3], filters [4], and sensors [5], along with many other uses. In [6]-[22], equivalent models of different types of two or 3-D SRRs (classical, cross embedded, U-shaped, etc.) have extensively been studied and

Manuscript received January 13, 2017; revised April 6, 2017; accepted April 11, 2017. Date of publication May 12, 2017; date of current version October 4, 2017.

B. Ozbey is with the Department of Electrical and Electronics Engineering, Bilkent University, Ankara TR-06800, Turkey (e-mail: ozbey@ee.bilkent.edu.tr).

A. Altintas is with the Department of Electrical and Electronics Engineering, Communications and Spectrum Management Research Center (ISYAM), Bilkent University, Ankara TR-06800, Turkey (e-mail: altintas@ee.bilkent.edu.tr).

H. V. Demir is with the Department of Electrical and Electronics Engineering and the Department of Physics, Institute of Materials Science and Nanotechnology (UNAM), Bilkent University, Ankara 06800, Turkey, and also with the School of Electrical and Electronic Engineering and the School of Physical and Mathematical Sciences, Nanyang Technological University, Singapore 639798 (e-mail: volkan@ @stanfordalumni.org).

V. B. Ertürk is with the Department of Electrical and Electronics Engineering, Bilkent University, Ankara 06800, Turkey (e-mail: vakur@ee.bilkent.edu.tr).

Color versions of one or more of the figures in this paper are available online at http://ieeexplore.iee.org.

Digital Object Identifier 10.1109/TMTT.2017.2699650 models based on different distributed line or lumped circuits were shown. In [18], analytical equivalent circuit models were proposed for SRRs and complementary SRRs, both for the isolated structures and for metamaterials, coupled to planar transmission lines to obtain effective negative constituent parameters. Equivalent models for structures incorporating SRRs coupled to transmission lines were further studied in detail [19]-[22].

Although SRRs have found a wide area of usage, they have been shown to have drawbacks for certain applications including sensing. Especially in the area of biosensing, in order to avoid the background absorption of soft tissue, a lower operating frequency is required along with a compact size for the sensor to be able to be placed in vivo [23]. The size of the conventional SRRs proves to be too large at lower frequencies for this purpose. Besides, in all sensing applications, an increased level of sensitivity is desired. Therefore, a new type of SRR was proposed in [23], called nested SRRs (NSRRs), which offer both a more compact size and a better sensitivity compared with classical SRRs. In this structure, there exist a number of parallel strip pairs that are connected from one side but symmetrically separated from the other by a gap between every pair. Each strip forms a path with the uppermost strip that is split by this gap, and therefore, the whole structure can be considered as a combination of nested split rings. The smaller size of the NSRR becomes possible via an increase in the number of metal strips, which in turn increases the overall capacitance and inductance of the structure and lowers the resonance frequency. Also, by the increased number of gaps, a high-Q resonator characteristic is achieved, bringing in better sensitivity and resolution. Although originally proposed for biosensing, the NSRRs have since been adapted to a diverse range of applications because of these advantages. The NSRR structure was employed in different antenna designs in microwave and optical frequencies [24]-[26]. A compact low-phase noise oscillator with superior harmonic suppression characteristics was developed with NSRR geometry [27]. Additionally, a compact NSRR-based filter [28] and an NSRR microwave thin-film sensor [29] were shown. The NSRR structure was further modified for displacement and strain sensing by splitting it into two mechanically independent halves and electrically connecting these two parts by a thin wire [30]. This modified NSRR structure was demonstrated to provide significant advantages in structural health monitoring, where wireless, passive, and compact sensors with the capability of measuring very small displacements are in high demand [30]-[33]. Despite having the superiority over 
the conventional SRR geometry in terms of sensitivity and compactness and being increasingly utilized in different application areas, an equivalent circuit model, which can explain the operation of the NSRR, has not been covered in literature. Such an equivalent model is required for better analysis and intuition of the physics of these structures. Furthermore, such a model is functional in facilitating the design of these complex structures, which normally employ a large number of strips and gaps. Finally, the applicability of the NSRR model to other SRR types would be easier (since NSRR is generally more detailed and complex) after necessary modifications.

In this paper, an equivalent circuit-based model of the NSRR is presented. The proposed circuit model is adaptable to both the classical and the modified NSRR structures. The model parameters originate from capacitances and inductances of simple shapes such as a piece of planar transmission line or two parallel wires, which are combined here to come up with the working principle of the whole NSRR. Therefore, rather than curve fitting to numerical or experimental results, the element values are derived from the physics of each structure, which is an important point to emphasize. Here, the NSRR structure is treated as a combination of strips and gaps between them, and the electrical and magnetic interactions of each strip with all the others are taken into account. The proposed model is able to adapt to the variation of different design parameters, with which the resonance frequency of the structure can be modified. Apart from the equivalent circuit model, another approach for modeling the NSRR is to treat each NSRR half as a multiconductor transmission line by setting the ground plane height relatively high to avoid coupling. On the other hand, this approach leads to less accurate results since the effects of the mutual inductances between the strips are not taken into account. In Section II, the geometries of the classical and modified NSRR are explained. In Section III, all model parameters are introduced and discussed in detail. In addition, formulas required for finding each parameter are derived. In Section IV, the methodology for the validation of the model results is described through a discussion of the simulation and measurement setups. In Section V, the outputs of the equivalent circuit model are compared with the results obtained from the measurements and full-wave simulations performed by CAD packages. Finally, conclusions derived from the study are discussed in Section VI.

\section{Classical And Modified NSRR Geometries}

The NSRR geometry modified for sensing the displacement in the direction of the strips is shown in Fig. 1. The whole structure consists of metallic strips printed on a dielectric substrate. Here, the edge-to-edge distance between two separated and mechanically independent parts of the structure is denoted by $d$. Likewise, $l$ represents the length of the thin wire used to electrically short the uppermost strip. It is noted that in the classical NSRR geometry, the uppermost strip is continuous and there is no wire, making the separation between strip pairs $d_{0}$, which is the initial separation (see Fig. 1). Hence, the classical NSRR is a special case of the modified NSRR structure, which is obtained by setting $d=0$ and $l=0$.

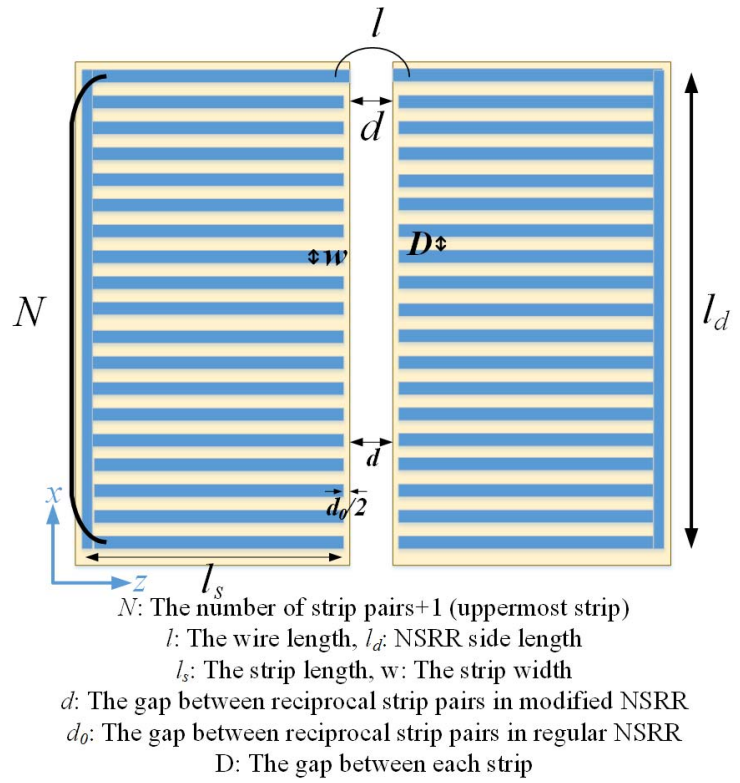

Fig. 1. NSRR geometry modified for displacement sensing. The structure is transformed to the classical NSRR geometry when $d=0$ and $l=0$ (and hence the uppermost strip is continuous).

In the modified NSRR geometry, an additional versatility is introduced by $d$ and $l$, which can be used for modifying the resonance frequency $f_{\text {res }}$ of the whole NSRR structure even after its fabrication. When a mechanical effect leads to an elongation or deformation of the structure on which the NSRR sensor is placed along one direction, this leads to a change of the overall capacitance and/or inductance of the sensor, resulting in a shift of $f_{\text {res }}$. This shift can be characterized by extraction of a $d-f_{\text {res }}$ curve for different media mimicking the environment in which the sensor is going to be placed. The measurement of displacement then becomes possible using the $d-f_{\text {res }}$ curve for transformation from the measured frequency change into the absolute $d$ value. Similarly, for the classical NSRR geometry, the variation of $f_{\text {res }}$ with the induced strain is tracked. $N, l_{d}, D$, and $w$ and $l_{s}$ shown in Fig. 1 denote the number of the parallel strip pairs (including the uppermost continuous strip), the NSRR side length, the gap between the adjacent strips, and the width and length of a strip, respectively. Especially $N$ is important for determining $f_{\text {res }}$ before the fabrication of the structure, while other parameters play roles in determination of the structure capacitances and inductances.

\section{Equivalent Circuit Model}

The proposed equivalent circuit model of NSRR is a representation that is valid only around the fundamental resonance frequency, which is obtained when the NSRR is in its fundamental mode of operation. This mode can be considered as a TE mode, in which the NSRR is excited by a plane wave in horizontal polarization, i.e., when the incident $E$ field is along the direction of the strips and gaps. In order to explain higher order modes, approaches different than the proposed model would be necessary, which are out of the scope of this paper. In the model, the NSRR comprises $N$ stages, each of which represents an opposing strip pair. There 




Fig. 2. Proposed equivalent circuit model for NSRRs showing the parameters of the model.

exist four independent lumped parameters in the model, two capacitances $\left(C_{s}\right.$ and $\left.C_{\text {gap }}\right)$ and two inductances $\left(L_{s}\right.$ and $\left.L_{\text {wire }}\right)$. $L_{\text {wire }}$ is present only for the modified NSRR geometry, and it is replaced by a short in the classical structure. The resistive or dielectric losses are ignored since they are too low to be considered at the operation frequency. The details regarding these parameters are as follows.

1) $C_{s}$ : This is the capacitance between two coplanar parallel strips. Therefore, the number of $C_{s}$ is $N-1$ for each of the two mechanically separated NSRR parts. $C_{s}$, along with the inductance of each strip $L_{s}$, are divided into $m$ segments as shown in Fig. 2. The reason for this is the following: when $C_{s}$ and $L_{s}$ are considered as the parameters of a lossless planar transmission line [e.g., coplanar stripline (CPS)], more accurate results are obtained through expressing them as distributed parameters instead of single lumped elements. This way, the structure resembles the equivalent circuit model of a lossless transmission line. The value of the capacitance of each segment from $C_{s, 1}$ to $C_{s, m}$ is simply assumed to be equal, and is given by

$$
C_{s, 1}=C_{s, 2}=\cdots=C_{s, m}=\frac{C_{s}}{m} .
$$

Several approaches can be utilized for the calculation of $C_{s}$. In this paper, each parallel strip pair will be treated as a CPS, from which the line capacitance can be calculated as $C_{s}$. The parallel strips are shorted from one side, but this does not constitute a problem since a shorted CPS can still be modeled as a transmission line without a discontinuity [34]. A single-layer CPS illustration is shown in Fig. 3. Using conformal mapping, the per-unit-length capacitance of such a transmission line, $C_{s, \mathrm{cps}}$, is given as [35], [36]

$$
C_{s, \mathrm{cps}}=\epsilon_{r, \mathrm{eff}}^{\mathrm{cps}} \epsilon_{0} \frac{K(k)}{K\left(k^{\prime}\right)}
$$

where $\epsilon_{r \text {,eff }}^{\mathrm{cps}}$ is the effective relative permittivity of the CPS, $\epsilon_{0}$ is the free-space permittivity, and $K$ denotes the complete elliptical integral of the first kind. The arguments $k$ and $k^{\prime}$ are functions of the physical parameters of the line and are given as

$$
k=\sqrt{1-\left(\frac{a}{b}\right)^{2}}
$$

and

$$
k^{\prime}=\sqrt{1-k^{2}}=\frac{a}{b}
$$

where $a$ and $b$ are the half of the inner and outer edgeto-edge distances of the two plate conductors of the CPS geometry, respectively, as shown in Fig. 3. The effective relative permittivity of the CPS $\epsilon_{r, \text { eff }}^{\mathrm{cps}}$ is found as [35], [36]

$$
\epsilon_{r, \mathrm{eff}}^{\mathrm{cps}}=1+\frac{1}{2}\left(\epsilon_{r}-1\right) \frac{K(k) K\left(k_{1}^{\prime}\right)}{K\left(k^{\prime}\right) K\left(k_{1}\right)}
$$

where $\epsilon_{r}$ is the relative permittivity of the substrate, and $k_{1}$ and $k_{1}^{\prime}$ are defined as

$$
k_{1}=\sqrt{1-\frac{\sinh ^{2}(\pi a / 2 h)}{\sinh ^{2}(\pi b / 2 h)}}
$$

and

$$
k_{1}^{\prime}=\sqrt{1-k_{1}^{2}}
$$

where $h$ is the thickness of the substrate as shown in Fig. 3 . By comparing Fig. 3 with Fig. 1, one can deduce that in the formulas, $a=D / 2$ and $b=(2 w+D) / 2$, where $w$ and $D$ are the strip width and the edge-to-edge gap between two coplanar strips, respectively, as shown in Fig. 1. An NSRR structure shown in [30], which is designed to operate at around $435 \mathrm{MHz}$ for displacement sensing in structural health monitoring, has the following parameters: $N=29, w=0.800 \mathrm{~mm}$, $D=0.800 \mathrm{~mm}, h=0.508 \mathrm{~mm}, l_{s}=21.6 \mathrm{~mm}$, and $\epsilon_{r}=3.2$. For this structure, $\epsilon_{r, \text { eff }}^{\mathrm{cps}}$ is calculated as 1.67. It should be noted that $\epsilon_{r, \text { eff }}^{\mathrm{cps}}$ is only a function of the physical dimensions and 


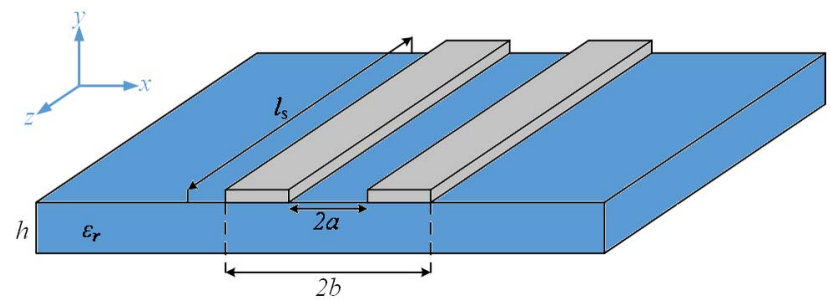

Fig. 3. Schematic of the parameters of a single-layer CPS.

substrate dielectric constant, and is independent of $N$. Then, the undistributed capacitance $C_{s}$ [given in (1)] is found as

$$
C_{s}=C_{s, \mathrm{cps}} l_{s}=0.524 \mathrm{pF} \text {. }
$$

This value is further divided into $m$ in circuit analysis.

2) $L_{s}$ : This is the total average inductance of each one of the strips. For more accuracy, similar to $C_{s}, L_{s}$ is also divided into $m$ segments (shown in Fig. 2) as in the lossless transmission line model. The inductance of each segment from $L_{s, 1}$ to $L_{s, m}$ is again assumed to be equal, and is given by

$$
L_{s, 1}=L_{s, 2}=\cdots=L_{s, m}=\frac{L_{s}}{m} .
$$

$L_{s}$ is composed of three components. The first component is the self-inductance of each strip, $L_{s, \text { self. The second one is }}$ the effect of the average mutual inductances coming from the strips parallel to a particular strip, denoted by $M$. The final component is the effect of the average mutual inductance of a strip with the strips that lie at the other half of the NSRR, and is denoted by $M_{r}$. The calculation methods for these components are described below.

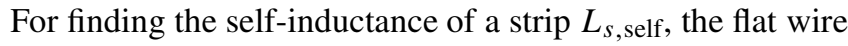
or ribbon inductance formula can be utilized, and is given in [34] and [37] as

$$
L_{s, \text { self }}=2 l_{s}\left[\ln \left(\frac{2 l_{s}}{w+t}\right)+0.5+0.2235\left(\frac{w}{l_{s}}\right)\right]
$$

where $t$ is the metal thickness and can be ignored in our study since the operation frequency is too low to include its effect. The other two parameters $l_{s}$ and $w$ are shown in Fig. 1.

$M$ and $M_{r}$, which are the mutual inductance components of $L_{s}$, should normally be treated by introducing a polarity to mutual inductive coupling of the strips with each other. In other words, the interaction of each strip with all the remaining ones should be taken into account via transformers with a polarity, which defines the directions and the magnitudes of the voltages due to these currents at each node. However, this approach would be problematic since $N$ is generally high, leading to a highly complicated network. In the simulations where the NSRR probe is illuminated by a plane wave, it is observed that the induced currents at all strips ( $I$ 's) are equal in direction and magnitude, which is also reasonable due to the symmetry of the structure. Therefore, the mutual inductances can be considered as series to the strip inductances, and $L_{s}$ can simply be taken as the addition of the three components, i.e., $L_{s}=L_{s, \text { self }}+M+M_{r}$.

$M$, which is the total mutual inductance of the strips parallel to it, can be calculated by employing the coplanar parallel

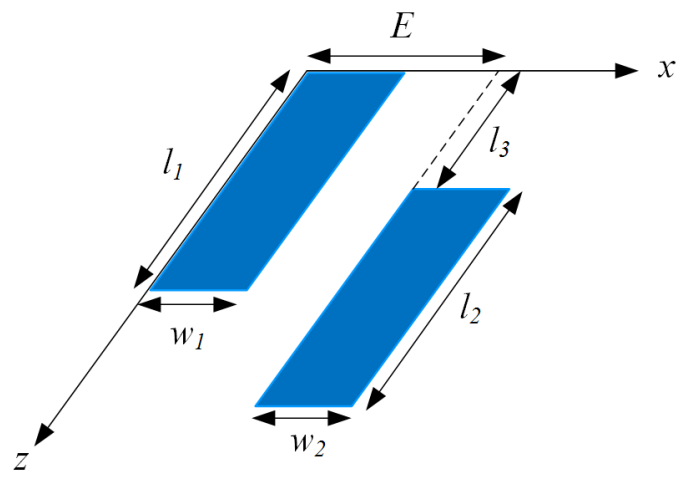

Fig. 4. Diagram showing the geometry for calculating mutual inductance between two parallel thin tapes.

thin tape mutual inductance formulation given in [38] for the corresponding geometry shown in Fig. 4. The formulation is as follows:

$$
\begin{aligned}
& M(E) \\
& =\frac{0.001}{w_{1} w_{2}}\left[\left[\left(\frac{x^{2} z}{2} \ln \left(z+\sqrt{x^{2}+z^{2}}\right)\right.\right.\right. \\
& +\frac{x z^{2}}{2} \ln \left(x+\sqrt{x^{2}+z^{2}}\right)-\frac{1}{6}\left(x^{2}+z^{2}\right) \\
& \left.\left.\left.\times \sqrt{x^{2}+z^{2}}\right)\right]_{E+w_{2}-w_{1}, E}^{E-w_{1}, E+w_{2}}(x)\right]_{l_{3}+l_{2}-l_{1}, l_{3}}^{l_{3}-l_{1}, l_{3}+l_{2}}(z)
\end{aligned}
$$

where

$$
\left[[f(x, z)]_{q_{2}, q_{4}}^{q_{1}, q_{3}}(x)\right]_{s_{2}, s_{4}}^{s_{1}, s_{3}}(z)=\sum_{i=1}^{4} \sum_{k=1}^{4}(-1)^{i+k} f\left(q_{i}, s_{k}\right)
$$

with $l_{1}$ and $l_{2}$ and $w_{1}$ and $w_{2}$ are the lengths and the widths of the strips, respectively, while $l_{3}$ is the shift of the second strip with respect to the first strip in the $z$-axis, as shown in Fig. 4. Here, $M$ is in microhenries.

In (11), $E$ is the outer-to-inner edge distance between the wires along the $x$-axis. In order to calculate $M$ for a strip, the mutual inductances coming from each parallel strip should be combined by setting $E_{i}=2-D, 4-D, 6-D, \ldots$, where $E_{i}$ represents the distance between the middle section of the first strip and that of a strip parallel to the first strip (see Fig. 4). The reason for this can be explained as follows: between two adjacent parallel strips, $E_{1}=w+D$. However, in the NSRR design, $w$ is generally set equal to $D$. Therefore, $E_{1}=w+D=2-D$, whereas for second adjacent strips, $E_{2}=D+w+D+w=4-D$, and so on. Hence, the total mutual inductance can be calculated using (11) with a changing $E$ for the contribution of each strip, setting $l_{3}=0$. On the other hand, one should also consider the magnetic fields that cancel each other for a strip on the NSRR structure. In Fig. 5, the calculation of the total $M$ is shown for $N=3,4,5$. As mentioned before, an equal current of $I$ is assumed to be induced at every strip. Then, under the equal current assumption, the magnetic fields due to $I$ s from symmetric up and down strips cancel each other for a specific 


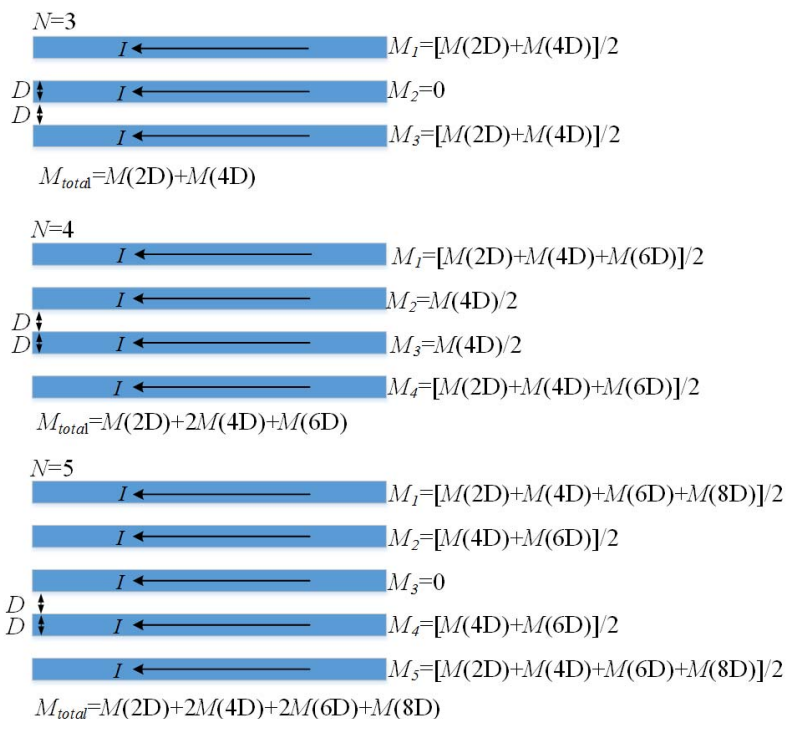

Fig. 5. Calculation of the mutual inductance $M_{\text {total }}$ between parallel strips for $N=3,4,5$.

strip. For instance, in Fig. 5, for $N=3$, the mutual inductance at the center strip $M_{2}$ is zero due to cancellation of fields. However, for $M_{1}$ and $M_{3}$, contributions from the strips, which are $2=D$ and $4=D$ away, are included. The total mutual inductance $M_{\text {total }}$ is then the sum of $M_{1} / 2, M_{2} / 2$, and $M_{3} / 2$, where division by 2 is to avoid including the same mutual inductance twice. This reasoning is valid for any $N$, but for even $N$, zero $M$ is not produced for any strip. The formulations of $M$ for even and odd $N$ are as follows:

$$
\begin{aligned}
\text { Odd } N: M_{\text {total }}= & \sum_{k=2}^{\frac{N+1}{2}}(k-1) M[(2 k-2) D] \\
& +\sum_{k=\frac{N+3}{2}}^{N}(N-k+1) M[(2 k-2) D] . \\
\text { Even } N: M_{\text {total }}= & \sum_{k=2}^{\frac{N}{2}+1}(k-1) M[(2 k-2) D] \\
& +\sum_{k=\frac{N}{2}+2}^{N}(N-k+1) M[(2 k-2) D] .
\end{aligned}
$$

In this formulation, $M_{\text {total }}$ gives the sum of all mutual inductances from all strips in parallel. However, the effect of $M_{\text {total }}$ has to be squeezed inside a single strip via $L_{S}$ in the equivalent circuit model. In order to reflect it on $L_{s}$ as an average value, it is assumed that $M_{\text {total }}$ is distributed to every strip equally, and thus should be divided by $N$

$$
M=\frac{M_{\mathrm{total}}}{N} .
$$

For a hypothetical case where $N \rightarrow \infty, M_{\text {total }}$ would be distributed to every strip exactly equally. Since generally a high number of $N$ is of interest for the NSRR geometry, this condition is approached, making the assumption of equal distribution of mutual inductances a plausible one (especially for high $N$ ).

$M_{r}$ is the mutual inductance of each strip with all opposing strips, i.e., the strips that stay on the other mechanically separated NSRR half. For the calculation of $M_{r},(11)$ can again be used with the exception that $l_{3}$ is now not 0 but a value that changes with $d$, where $d$ is the edge-to-edge distance between two separated parts of the modified NSRR (as shown in Fig. 1). In order to obtain $M_{r \text {,total }}$, the mutual inductance of a strip with all opposing strips should be combined in a fashion similar to the calculation of $M_{\text {total }}$. For every strip pair, (11) should be used by setting $l_{3}=l_{s}+d+d_{0}$. However, for the calculation of $M_{r, \text { total }}$, different from the algorithm described in Fig. 5, the contribution of the uppermost strip is not included since it is continuous and does not have a gap with the opposing strips. Also, mutual inductance of a strip with the directly opposing one is not considered since the magnetic field is negligible along the axis of the strip. The cancellation of fields again takes place for even $N$ for the middle strip excluding the uppermost strip. The formulation of $M_{r}$ for odd and even $N$ is then as follows:

$$
\begin{aligned}
\text { Odd } N: M_{r, \text { total }}= & \sum_{k=3}^{\frac{N+3}{2}}(k-2) M[(2 k-4) D] \\
& +\sum_{k=\frac{N+5}{2}}^{N}(N-k+1) M[(2 k-4) D] \\
\text { Even } N: M_{r, \text { total }}= & \sum_{k=3}^{\frac{N}{2}+1}(k-2) M[(2 k-4) D] \\
& +\sum_{k=\frac{N}{2}+2}^{N}(N-k+1) M[(2 k-4) D]
\end{aligned}
$$

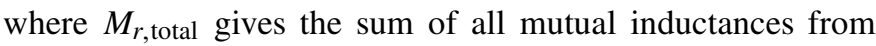
the opposing strips. By the same reasoning for $M_{\text {total }}$, in order to reflect $M_{r \text {,total }}$ on $L_{s}$, which is the inductance of a single strip, $M_{r \text {,total }}$ should be divided by $N$

$$
M_{r}=\frac{M_{r, \text { total }}}{N} .
$$

After calculation of the self-inductance of each strip and the mutual inductances coming from the parallel and opposing strips, the average inductance reflected in $L_{S}$ can be written as

$$
L_{s}=L_{s, \text { self }}+M+M_{r} .
$$

For the application of displacement sensing, $d$ changes with a mechanical effect causing a relative displacement in that direction, and hence $M_{r}$ and $L_{s}$ become dependent on $d$.

3) $C_{\text {gap }}$ : This is the capacitance between an opposing strip pair. Hence, the number of $C_{\text {gap }}$ in the model is $N-1$. Again, different approaches can be taken for finding $C_{\text {gap }}$. The microstrip discontinuity formulas available in [39] and [40] do not apply due to the presence of the bottom conductor. Here, the best approach seems to be modeling the opposing strip pair as a piece of a thick and very short CPS, where $w$ is the 
TABLE I

ChANGe of $L_{\text {WIRE }}$ With $l\left(r_{w}=0.1 \mathrm{~mm}\right)$

\begin{tabular}{|c|c|c|c|c|c|}
\hline$l(\mathrm{~cm})$ & 1.00 & 3.00 & 5.00 & 7.00 & 9.00 \\
\hline$L_{\text {wire }}(\mathrm{nH})$ & 9.10 & 33.9 & 61.6 & 90.9 & 121 \\
\hline
\end{tabular}

length of the line and $2 a=d+d_{0}$ and $2 b=2 l_{s}+d+d_{0}$, when the notation in Fig. 3 is employed. Then, $C_{\text {gap }}$ is given by

$$
C_{\text {gap }}=\epsilon_{r, \text { eff }}^{\mathrm{cps}} \epsilon_{0} \frac{K(k)}{K\left(k^{\prime}\right)} w
$$

where

$$
k=\sqrt{1-\left(\frac{d+d_{0}}{2 l_{s}+d+d_{0}}\right)^{2}}
$$

and

$$
k^{\prime}=\sqrt{1-k^{2}}=\frac{d+d_{0}}{2 l_{s}+d+d_{0}} .
$$

The effective relative permittivity of the CPS $\epsilon_{r, \text { eff }}^{\mathrm{cps}}$ is again taken as 1.67 from (5) for the set of physical parameters mentioned before. Similar to $M_{r}, C_{\text {gap }}$ is also a function of $d$. For the application of displacement sensing, the effect of the decrease in $C_{\text {gap }}$ becomes dominant and leads to an increase in $f_{\text {res }}$.

4) $L_{\text {wire: }}$ The uppermost strip is continuous in NSRR geometry, and this can be achieved via a thin wire when the whole structure is symmetrically split into two halves. As previously mentioned, another function of this shorting wire is to help tuning $f_{\text {res }}$ by assigning different values for $l$, the wire length. $L_{\text {wire }}$ does not exist for the classical NSRR (when the structure is not split into two parts), in which case it can be replaced by just a short. In the literature, there are several variations of the formula for the self-inductance of a thin wire, all of which yield very approximate results for $L_{\text {wire }}$. Here, the wire inductance is found as [41]

$$
L_{\text {wire }}=2 l\left[\ln \left(\frac{2 l}{r_{w}}\right)-\frac{3}{4}\right](\mathrm{nH})
$$

where $r_{w}$ is the radius of the wire. $L_{\text {wire }}$ is shown for several values of $l$ in Table I for $r_{w}=0.100 \mathrm{~mm}$. As mentioned before, mutual impedance due to $L_{\text {wire }}$ is not considered.

The equivalent circuit model parameter values obtained for six different $N$ between 5 and 29 as well as for $d=0$ and $d=5 \mathrm{~mm}$ are given in Table II for the following set of physical dimensions: $w=0.800 \mathrm{~mm}, D=0.800 \mathrm{~mm}$, $d_{0}=0.800 \mathrm{~mm}, l_{s}=21.6 \mathrm{~mm}, h=0.508 \mathrm{~mm}$, and $\epsilon_{r}=3.2$. It should be noted that $C_{s}, C_{\mathrm{gap}}$, and $L_{s, \text { self }}$ are independent of $N$, whereas the mutual inductances $M$ and $M_{r}$ change with $N$. $C_{\text {gap }}$ and $M_{r}$ are the only two parameters that are functions of $d$. Only $L_{\text {wire }}$ is a function of $l$, of which variation is shown in Table I and is not repeated here. In addition, the variations of the inductance parameters in the model ( $L_{s, \text { self }}, M, M_{r}$ and the total inductance $L_{S}$ ) with $N$ are shown in Fig. 6 . It can be observed in Fig. 6 that the slopes of $M$ and $M_{r}$ start to decrease as $N$ is increased, meaning that the addition of extra strips starts to be somehow ineffective for decreasing the resonance frequency above a certain $N$.

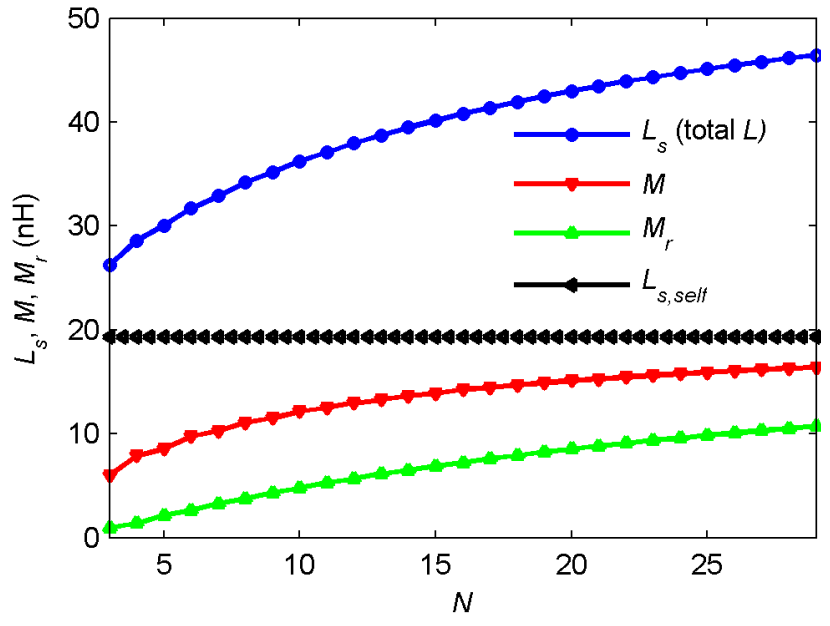

Fig. 6. Change of average strip inductance $L_{S}$ and its components $L_{s \text {, self, }}$ $M$, and $M_{r}$ with the number of strips $N$ for the classical NSRR geometry $(d=0$ and $l=0)$.

\section{Simulation and Measurement Setup}

\section{A. Simulation of the Model}

Simulations of the equivalent circuit model illustrated in Fig. 2 are carried out in Ansoft Designer. In the simulations, two high-impedance ports are defined at either side of the circuit so as not to disturb the resonator characteristics. In such a setup, the frequency of resonance $\left(f_{\text {res }}\right)$ can be determined by observing the transmission spectrum, i.e., tracking the position of the dip frequency of the magnitude of $S_{21}$ (the frequency where the phase crosses $0^{\circ}$ can also be used for this purpose). A typical $S_{21}$ magnitude and phase plot is shown in Fig. 7 for the classical NSRR geometry $(d=0$ and $l=0)$ when $N=29$. It can be observed that $f_{\text {res }}=535 \mathrm{MHz}$ for that particular case.

\section{B. Full-Wave Simulation Setup}

In order to verify the circuit model results, two sets of full-wave simulations are performed in CST Microwave Studio. In the first set of simulations, the NSRRs are excited by a plane wave in horizontal polarization ( $E$-field parallel to the orientation of the strips) [30]. The goal of this simulation is to see the effect of $N$ on the NSRR resonance frequency. The set of physical dimensions and electrical properties given in Table II is used for the simulated NSRR. The variation of $f_{\text {res }}$ obtained from the model for every $N$ from 3 to 29 is compared with the full-wave simulation results in Fig. 8. It should be noted that the structure takes a square shape only when $N=29$ with the given parameter set. In the simulations, as $N$ is increased, $w$ and $D$ are kept constant. Since $l_{s}$ also remains the same, the edge lengths take different values and the ratio of the higher to lower edge length is inversely proportional to $N$, becoming 1 only when $N=29$. In Fig. 8, the variation of $f_{\text {res }}$ is also shown when the number of line segments $m$ is set as 5, 10, and 15. It can be observed that the case when $m=5$ exhibits a relatively lower resonance frequency, but for higher $m, f_{\text {res }}$ does not vary much. Therefore, for the sake of accuracy and simplicity 
TABLE II

Equivalent Circuit Model Parameters for Different $N$ and $d$ Values $\left(w=0.800 \mathrm{~mm}, D=0.800 \mathrm{~mm}, d_{0}=0.800 \mathrm{~mm}, l_{s}=21.6 \mathrm{~mm}\right.$, $h=0.508 \mathrm{~mm}$, AND $\epsilon_{r}=3.2 ; C_{s}$ AND $C_{\mathrm{GAP}}$ ARE IN PiCOFARADS; $L_{s, \mathrm{SELF}}, M$, AND $M_{r}$ ARE IN NANOHENRIES; AND $d$ IS IN MiLLIMETERS)

\begin{tabular}{|c|c|c|c|c|c|c|c|c|c|}
\hline$N$ & $C_{s}$ & $C_{g a p}(d=0)$ & $C_{g a p}(d=5)$ & $L_{s, \text { self }}$ & $M$ & $M_{r}(d=0)$ & $M_{r}(d=5)$ & $L_{s}(d=0)$ & $L_{s}(d=5)$ \\
\hline 5 & 0.524 & 0.0431 & 0.0283 & 19.3 & 8.55 & 2.16 & 1.59 & 30.0 & 29.5 \\
10 & 0.524 & 0.0431 & 0.0283 & 19.3 & 12.1 & 4.75 & 3.75 & 36.2 & 35.2 \\
15 & 0.524 & 0.0431 & 0.0283 & 19.3 & 13.9 & 6.90 & 5.68 & 40.1 & 38.9 \\
20 & 0.524 & 0.0431 & 0.0283 & 19.3 & 15.1 & 8.52 & 7.23 & 43.0 & 41.7 \\
25 & 0.524 & 0.0431 & 0.0283 & 19.3 & 15.9 & 9.86 & 8.56 & 45.1 & 43.8 \\
29 & 0.524 & 0.0431 & 0.0283 & 19.3 & 16.4 & 10.7 & 9.45 & 46.5 & 45.2 \\
\hline
\end{tabular}


Fig. 7. Typical transmission spectrum plot obtained via the proposed equivalent circuit model, where the magnitude and phase of $S_{21}$ are shown. Here, $N=29, d=0$, and $l=0$ (classical NSRR geometry).

of the equivalent model, the results for $m=10$ are presented for the rest of the examples in this paper. Fig. 8 demonstrates that there is a correlation between the full-wave simulations and the proposed circuit model in terms of both the trend of variation and the absolute values of $f_{\text {res }}$. An important point is that the NSRR structure reduces to one of the rings of the well-known edge-coupled SRR geometry when $N=2$, which by itself is also used as a unit cell in metamaterials. This limiting case is important in terms of the adaptability of the model to other SRR types.

In the second set of full-wave simulations, the goal is to observe the effects of the change of $d$ and $l$ on the resonance frequency of the modified NSRR. In a practical sensing application, after the fabrication, $d$ and $l$ can be adjusted to determine $f_{\text {res }}$ before the NSRR is installed as a sensor. By the coupling between a transceiver antenna and the NSRR, the resonance of NSRR can be observed in the form of a peak at the reflection coefficient of the antenna [30], [31]. Therefore,



Fig. 8. Change of $f_{\text {res }}$ with $N$, shown for both equivalent circuit model and full-wave simulations. Here, $d=0$ and $l=0$.

in these simulations, the whole measurement setup is simulated including the antennas. In both measurements and simulations, $f_{\text {res }}$ is recorded and compared with the result obtained from the proposed equivalent circuit model. In the case where a sniffer coil is used for excitation, the NSRR resonance occurs at the off-resonance of the coil, and the coupling is lower compared with the antenna excitation. Therefore, the full-wave simulation for this case is not included. The results of these simulations are shown and discussed in Section V.

\section{Measurement Setup}

The variation of $f_{\text {res }}$ with the sensing and tuning parameters $d$ and $l$ is an important indicator of the accuracy of the model for the modified NSRR geometry. In order to compare the model results with those of the measurement, modified NSRRs are fabricated with the set of physical dimensions given in Table II for two different $N$ values: $N=10$ and $N=29$. The fabricated modified NSRRs are shown in Fig. 9. Two separate sets of mesurement data are taken with two different excitation sources: a microstrip single-slot antenna and a coil. As the first set of measurements, two single-slot microstrip antennas are designed at the resonance frequency range of the NSRRs with $N=10$ and $N=29$. With a sufficient bandwidth, it is possible to capture the shifting resonance frequency of the NSRR. The NSRR is placed within the near field of the antenna (at a distance smaller than the operation wavelength) in order to form a strong 




Fig. 9. Diagram of the measurement setup and the fabricated modified NSRR structures for $N=29$ and $N=10$.

electromagnetic coupling between the two structures, from which high-resolution and high-sensitivity sensing becomes possible. The NSRR orientation has to be such that the $E$-field is in the direction of the strips; no coupling is observed in the case of cross polarization. In the measurements, the antenna transmits the signal from the network analyzer (Agilent FieldFox N9915A) and collects back the scattered waves, as shown in Fig. 9. The distance between the antenna and the NSRR is set to $10 \mathrm{~cm}$. The setup employed to carry out the measurements is covered in detail in [30].

As the second set of measurements, the same procedure was repeated with a coil, called a sniffer coil, which can be used to pick up the resonance characteristics through inductive coupling (see Fig. 9). Unlike the antenna, the coil captures the NSRR resonance at its own off-resonance, where the NSRR resonance is observed as a notch at the coil spectrum. This second measurement forms an alternative to the antenna excitation, and is necessary to make sure that the frequency measured from the antenna via the electromagnetic coupling is very close to NSRR resonance frequency.

\section{RESULTS}

The measurement and full-wave simulation results (for the cases of antenna and plane wave excitation) are compared with those of the equivalent circuit model for two different NSRR structures with $N=10$ and $N=29$. The variation of $f_{\text {res }}$ with the sensing parameter $d$ is shown in Fig. 10 for an $l$ value of $4 \mathrm{~cm}$. As observed in Fig. 10, the resonance frequencies obtained by both the antenna and the coil excitations are close. The full-wave simulation results are shown for both the antenna excitation and the plane wave excitation. It is observed that the resonance frequencies obtained at the plane wave excitation are between the results of the equivalent circuit model and of the full-wave simulations with the antenna excitation. The difference between the simulation and measurement for antenna excitation is due to the difficulty of thoroughly mimicking the measurement setup. Factors such as the effect of the clutter (surrounding objects) or the orientation of the shorting wire of the NSRR play an important role in this difference. Despite these factors, it is seen that the model produces results that are close to the full-wave simulations and measurements. It is apparent that the agreement of the equivalent circuit model is better when $N=29$ in comparison with when $N=10$. As mentioned before, when $N=29$, a square-shaped structure

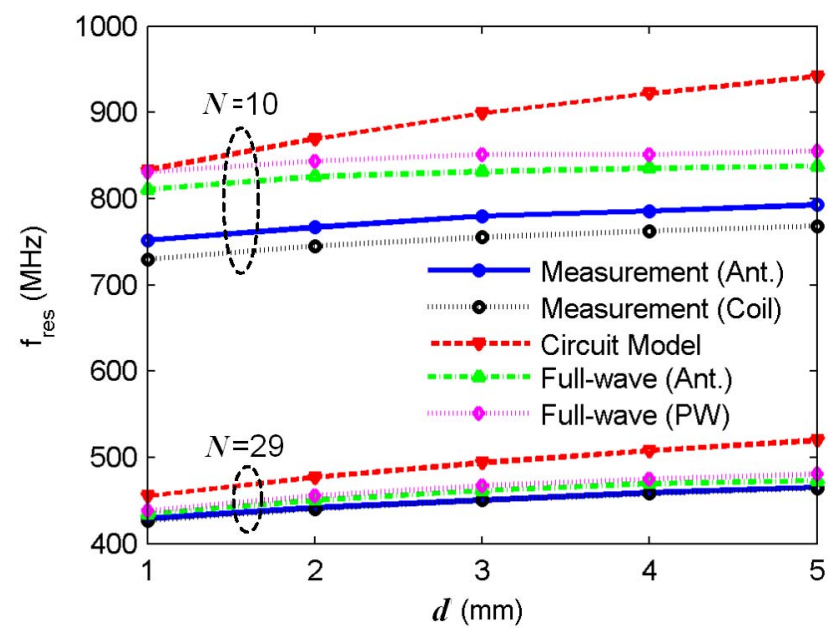

Fig. 10. Change of resonance frequency $f_{\text {res }}$ with displacement between the opposing strips $d$, which is obtained from the equivalent circuit model, compared with the results of measurement and full-wave simulation for the modified NSRR geometry. $l=4 \mathrm{~cm}$ for simulations and measurement.

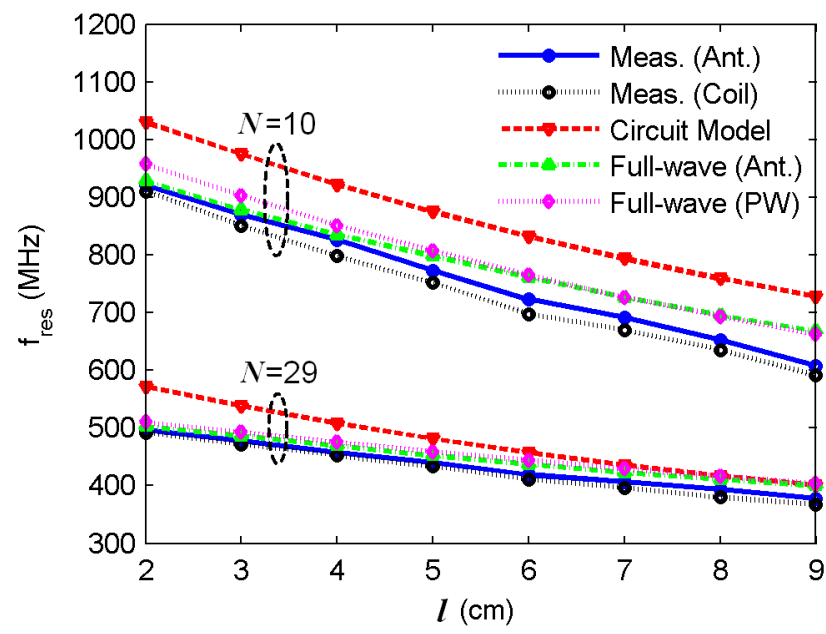

Fig. 11. Change of resonance frequency $f_{\text {res }}$ with shorting wire length $l$, which is obtained from the equivalent circuit model, compared with the results of measurement and full-wave simulation for the modified NSRR geometry. $d=4 \mathrm{~mm}$ for simulations and measurement.

is obtained with the used set of physical dimensions. However, for $N=10$, the result is a rectangle-shaped structure in which the edge where strips are elongated is much longer than the other edge. From Figs. 10 and 11, as well as from the variation of the model resonance frequency with $N$ shown in Fig. 8, it can be concluded that the model works better for a bigger size and a square-shaped structure in comparison with a rectangular-shaped structure.

The change of $f_{\text {res }}$ with the tuning parameter $l$ is also of interest for the modified NSRR geometry. In the experiments performed to characterize this change, the shorting wire length was changed from 2 to $9 \mathrm{~cm}$, while $d$ was kept constant at $4 \mathrm{~mm}$. The measurement results are compared with those of the equivalent circuit model in Fig. 11 again for $N=10$ and $N=29$. The trends of the variation of the measurement and the model resonance frequencies are observed to be in good agreement for $N=29$. For $N=10$, the agreement of model and measurement results can still be considered reasonable in 

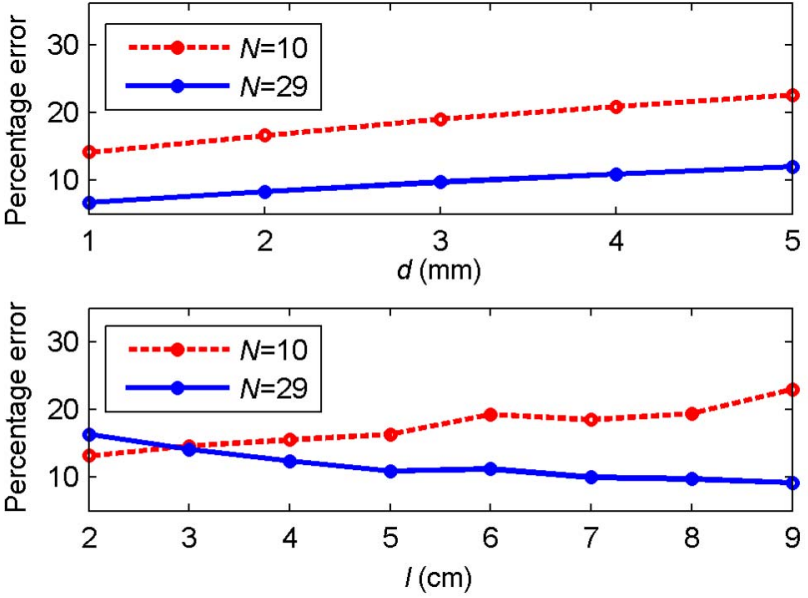

Fig. 12. Percentage error in $f_{\text {res }}$ at different values of $d$ and $l$, for $N=10$ and $N=29$.

terms of the trends of the curves despite the aforementioned disproportion of shape as $N$ is decreased. Although the measurement and full-wave results are not exactly on top of each other in Figs. 10 and 11, they are close and their trends are very similar. For $N=10$, the discrepancy between the fullwave and measurement results is higher, because the resonance frequency is higher. This can be observed in Fig. 12, where the percentage errors at different $d$ and $l$ values are shown for $N=10$ and $N=29$. The percentage error is defined as follows:

$$
\text { Percentage error }=\left|\frac{f_{\text {res,ecm }}-f_{\text {res,coil }}}{f_{\text {res,coil }}}\right| \times 100
$$

where $f_{\text {res,ecm }}$ and $f_{\text {res,coil }}$ are the resonance frequencies obtained from the equivalent circuit model and from the measurements with coil excitation, respectively. The coil measurement is taken as a reference to represent the worst case scenario (highest error value). For both parameters, the worst case error generally seems to be within the range 7\%-20\%.

The adaptability of the proposed model to the change in physical dimensions, such as the width and length of each strip, is also examined, and the results are given in Fig. 13. Unlike the case where the effect of $N$ was investigated on the resonance frequency, here the square shape of the classical NSRR structure is preserved in a given range of $l_{d}$, the NSRR side length, by varying both $w$ and $l_{s}$ to come up with each $l_{d}$ value. The results shown in Fig. 13 are for 7 values of $l_{d}$ changing from $11.4 \mathrm{~mm}$ to $79.8 \mathrm{~mm}$ in $11.4 \mathrm{~mm}$ steps for a structure with $N=29$. For these $l_{d}$ values, the strip widths are $w_{i}=0.2,0.4, \ldots, 1.4 \mathrm{~mm}$, and the strip lengths are $l_{s, i}=5.4,10.8, \ldots 37.8 \mathrm{~mm}$, where $i=1,2, \ldots, 7$. It should also be noted that $D_{i}=d_{0, i}=w_{i}$ at each case. In the graph, it can be observed that the model results are matched to those produced by the full-wave simulations when the NSRR shape is a square. The NSRRs are excited by a plane wave in the full-wave simulations. The agreement between the model results and the simulations is especially strong for larger physical dimensions. In fact, with the exception of the circular SRRs, an equilateral SRR geometry is almost always preferred as a unit cell in metamaterials or for other purposes



Fig. 13. Change of resonance frequency $f_{\text {res }}$ with the square NSRR side length $l_{d}$, which is obtained from the equivalent circuit model, compared with the result of the full-wave simulation for the classical geometry. Here, $w_{i}=0.2,0.4, \ldots, 1.4 \mathrm{~mm}$, and $l_{s, i}=5.4,10.8, \ldots 37.8 \mathrm{~mm}, i=1,2, \ldots, 7$.

in the literature. Hence, the agreement in Fig. 13 is significant, proving that the model is adaptable to a wideband range of $f_{\text {res }}$ from a few hundred megahertz to several gigahertz in practice.

\section{CONCLUSION}

In this paper, a rigorous and highly accurate equivalent circuit model is proposed and demonstrated for modeling NSRRs. In order to test the accuracy of the proposed equivalent circuit model, the change of resonance frequency with the variation of various design parameters is investigated for the classical and modified NSRR geometries, and the results that are obtained from the model are compared with those from the full-wave simulations and measurements. A good agreement is observed, demonstrating that the approach to the problem is correct. Two different sources are used as an excitation in the measurements: an antenna operating at its near field, which captures the NSRR response within its resonance bandwidth, and a sniffer coil, which tracks the NSRR resonance at its own off-resonance case. The equivalent circuit model results are observed to be especially accurate when the NSRR shape is square rather than rectangular, which is a much more common geometry. Through modification of parameters and employing the suitable formulas, the approach embraced here can be adapted to other types of planar metamaterial-inspired structures, mainly, other variants of SRRs. For example, as a limiting case, the classical NSRR geometry reduces to a onering edge-coupled SRR geometry. The fact that the inductance and capacitance values stem from the physics of the structure instead of curve fitting to numerical or experimental data, and the adaptability to a wide range of frequencies and physical dimensions shows that the proposed model offers a better and more efficient way of analysis and can act as preliminary design tool for the NSRR structures. However, due to a relatively high percentage error ranging from 7 to 20 , it would need to be improved in case the model is intended to be used for a very accurate design. 


\section{REFERENCES}

[1] J. B. Pendry, A. J. Holden, D. J. Robbins, and W. J. Stewart, "Magnetism from conductors and enhanced nonlinear phenomena," IEEE Trans. Microw. Theory Techn., vol. 47, no. 11, pp. 2075-2084, Nov. 1999.

[2] F. Martín, J. Bonache, F. Falcone, M. Sorolla, and R. Marqués, "Split ring resonator-based left-handed coplanar waveguide," Appl. Phys. Lett., vol. 83, no. 22, pp. 4652-4654, Nov. 2003.

[3] K. B. Alici and E. Ozbay, "Electrically small split ring resonator antennas," J. Appl. Phys., vol. 101, no. 8, p. 083104, Apr. 2007.

[4] W. Che, C. Li, K. Deng, and L. Yang, "A novel bandpass filter based on complementary split rings resonators and substrate integrated waveguide," Microw. Opt. Technol. Lett., vol. 50, no. 3, pp. 699-701, Mar. 2008.

[5] H.-J. Lee and J.-G. Yook, "Biosensing using split-ring resonators at microwave regime," Appl. Phys. Lett., vol. 92, no. 25, p. 254103, Jun. 2008.

[6] R. Marqués, F. Medina, and R. Rafii-El-Idrissi, "Role of bianisotropy in negative permeability and left-handed metamaterials," Phys. Rev. B, Condens. Matter, vol. 65, no. 14, p. 144440, Apr. 2002.

[7] G. V. Eleftheriades, O. Siddiqui, and A. K. Iyer, "Transmission line models for negative refractive index media and associated implementations without excess resonators," IEEE Microw. Wireless Compon. Lett., vol. 13, no. 2, pp. 51-53, Feb. 2003.

[8] H. Chen, L. Ran, J. Huangfu, T. M. Grzegorczyk, and J. A. Kong, "Equivalent circuit model for left-handed metamaterials," J. Appl. Phys., vol. 100 , no. 2, p. 024915 , Jul. 2006.

[9] L. J. Rogla, J. Carbonell, and V. E. Boria, "Study of equivalent circuits for open-ring and split-ring resonators in coplanar waveguide technology," IET Microw., Antennas Propag., vol. 1, no. 1, pp. 170-176, Feb. 2007.

[10] T. D. Corrigan, P. W. Kolb, A. B. Sushkov, H. D. Drew, D. C. Schmadel, and R. J. Phaneuf, "Optical plasmonic resonances in split-ring resonator structures: An improved LC model," Opt. Exp., vol. 16, no. 24 , pp. 19850-19864, Nov. 2008

[11] H. Bahrami, M. Hakkak, and A. Pirhadi, "Analysis and design of highly compact bandpass waveguide filter utilizing complementary split ring resonators (CSRR)," Prog. Electromagn. Res., vol. 80, pp. 107-122, 2008.

[12] S. Li, H.-W. Zhang, Q.-Y. Wen, Y.-S. Xie, D.-B. Tian, and Y.-X. Li, "Improved TL-RLC model for terahertz circular split-ring resonators," Appl. Phys. A, Solids Surf., vol. 100, no. 2, pp. 461-466, Aug. 2010.

[13] O. A. Safia, L. Talbi, and K. Hettak, "A new type of transmission linebased metamaterial resonator and its implementation in original applications," IEEE Trans. Magn., vol. 49, no. 3, pp. 968-973, Mar. 2013.

[14] A. Ebrahimi, W. Withayachumnankul, S. F. Al-Sarawi, and D. Abbott, "Dual-mode behavior of the complementary electric-LC resonators loaded on transmission line: Analysis and applications," J. Appl. Phys., vol. 116, no. 8, Aug. 2014, Art. no. 083705.

[15] T. Zhang, W. Xiong, B. Zhao, J. Shen, C. Qiu, and X. Luo, "Equivalent circuit analysis of 'U'-shaped split ring resonators," J. Mod. Opt., vol. 62, no. 11, pp. 901-907, Jun. 2015.

[16] A. A. Abduljabar, X. Yang, D. A. Barrow, and A. Porch, "Modelling and measurements of the microwave dielectric properties of microspheres," IEEE Trans. Microw. Theory Techn., vol. 63, no. 12, pp. 4492-4500, Dec. 2015.

[17] R. Bojanic, V. Milosevic, B. Jokanovic, F. Medina-Mena, and F. Mesa, "Enhanced modelling of split-ring resonators couplings in printed circuits," IEEE Trans. Microw. Theory Techn., vol. 62, no. 8, pp. 1605-1615, Aug. 2014.

[18] J. D. Baena et al., "Equivalent-circuit models for split-ring resonators and complementary split-ring resonators coupled to planar transmission lines," IEEE Trans. Microw. Theory Techn., vol. 53, no. 4, pp. 1451-1461, Apr. 2005.

[19] A. L. Borja, A. Belenguer, J. Cascon, H. Esteban, and V. E. Boria, "Wideband passband transmission line based on metamaterial-inspired CPW balanced cells," IEEE Antennas Wireless Propag. Lett., vol. 10, pp. 1421-1424, 2011

[20] J. Naqui, M. Duran-Sindreu, and F. Martín, "Modeling split-ring resonator (SRR) and complementary split-ring resonator (CSRR) loaded transmission lines exhibiting cross-polarization effects," IEEE Antennas Wireless Propag. Lett., vol. 12, pp. 178-181, 2013.

[21] J. Naqui, L. Su, J. Mata, and F. Martín, "Analysis of transmission lines loaded with pairs of coupled resonant elements and application to sensors," J. Magn. Magn. Mater, vol. 383, pp. 144-151, Jun. 2015
[22] L. Su, J. Naqui, J. Mata-Contreras, and F. Martín, "Modeling metamaterial transmission lines loaded with pairs of coupled split-ring resonators," IEEE Antennas Wireless Propag. Lett., vol. 14, pp. 68-71, 2015.

[23] R. Melik et al., "Nested metamaterials for wireless strain sensing," IEEE J. Sel. Topics Quantum Electron., vol. 16, no. 2, pp. 450-458, Mar. 2010.

[24] S. Naoui, L. Latrach, and A. Gharsallah, "Nested metamaterials antenna for RFID traceability," Microw. Opt. Technol. Lett., vol. 56, no. 7, pp. 1622-1626, Jul. 2014.

[25] Y. Li, W. Li, and Q. Ye, "A compact UWB antenna with dual band-notch characteristics using nested split ring resonator and stepped impedance resonator," Microw. Opt. Technol. Lett., vol. 55, no. 12, pp. 2827-2830, Dec. 2013.

[26] V. T. Kilic, V. B. Erturk, and H. V. Demir, "Optical antenna of combshaped split ring architecture for increased field localization in NIR and MIR," Opt. Exp., vol. 21, no. 24, pp. 29455-29461, Dec. 2013.

[27] Y. Liu, N. Xie, X. Tang, and F. Xiao, "A compact low-phase noise oscillator with superior harmonic suppression characteristics based on novel nested split-ring resonator (NSRR)," Int. J. Microw. Wireless Technol., vol. 8, no. 8, pp. 1155-1161, Dec. 2016.

[28] Y. Liu, X. Tang, Z. X. Zhang, and X. L. Huang, "Novel nested splitring-resonator (SRR) for compact filter application," Prog. Electromagn. Res., vol. 136, pp. 765-773, 2013.

[29] X.-J. He, L. Qiu, Y. Wang, Z.-X. Geng, J.-M. Wang, and T.-L. Gui, "A compact thin-film sensor based on nested split-ring-resonator (SRR) metamaterials for microwave applications," J. Infr., Millim., Terahertz. Waves, vol. 32, no. 7, pp. 902-913, Jul. 2011.

[30] B. Ozbey et al., "Wireless displacement sensing enabled by metamaterial probes for remote structural health monitoring," Sensors, vol. 14, no. 1, pp. 1691-1704, Jan. 2014.

[31] B. Ozbey, H. V. Demir, O. Kurc, V. B. Erturk, and A. Altintas, "Wireless measurement of elastic and plastic deformation by a metamaterial-based sensor," Sensors, vol. 14, no. 10, pp. 19609-19621, Oct. 2014.

[32] B. Ozbey, H. V. Demir, O. Kurc, V. B. Erturk, and A. Altintas, "Wireless sensing in complex electromagnetic media: Construction materials and structural monitoring," IEEE Sensors J., vol. 15, no. 10, pp. 5545-5554, Oct. 2015.

[33] B. Ozbey, V. B. Erturk, H. V. Demir, A. Altintas, and O. Kurc, "A wireless passive sensing system for displacement/strain measurement in reinforced concrete members," Sensors, vol. 16, no. 4, p. 496, Apr. 2016.

[34] B. C. Wadell, Transmission Line Design Handbook. Boston, MA, USA: Artech House, May 1991

[35] E. Chen and S. Y. Chou, "Characteristics of coplanar transmission lines on multilayer substrates: Modeling and experiments," IEEE Trans. Microw. Theory Techn., vol. 45, no. 6, pp. 939-945, Jun. 1997.

[36] R. N. Simons, Coplanar Waveguide Circuits, Components, and Systems, 1st ed. New York, NY, USA: Wiley, Apr. 2001.

[37] F. E. Terman, Radio Engineers' Handbook, 1st ed. New York, NY, USA: McGraw-Hill, 1943.

[38] C. Hoer and C. Love, Exact Inductance Equations for Rectangular Conductors With Applications to More Complicated Geometries. Boulder, CO, USA: NBS, 1965

[39] M. Kirschning, R. H. Jansen, and N. H. L. Koster, "Measurement and computer-aided modeling of microstrip discontinuities by an improved resonator method," in IEEE MTT-S Int. Microw. Symp. Dig., May/Jun. 1983, pp. 495-497.

[40] K. C. Gupta, R. Garg, I. Bahl, and P. Bhartia, Microstrip Lines and Slotlines, 2nd ed. Boston, MA, USA: Artech House, Feb. 1996.

[41] F. W. Grover, Inductance Calculations: Working Formulas and Tables. North Chelmsford, MA, USA: Courier Corporation, 2004.

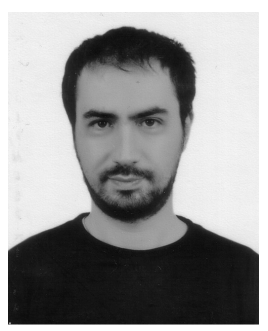

Burak Ozbey (M'16) received the B.S., M.S., and $\mathrm{Ph} . \mathrm{D}$. degrees in electrical and electronics engineering from Bilkent University, Ankara, Turkey, in 2008, 2011, and 2016, respectively.

$\mathrm{He}$ is currently a Post-Doctoral Researcher with the Electrical and Electronics Engineering Department, Bilkent University. His current research interests include electromagnetic theory, microwave circuits, structural health monitoring, and the design and testing of wireless RF sensors.

Dr. Ozbey was a recipient of the 2016 Leopold B. Felsen Award for Excellence in Electromagnetics. He was selected as a Fulbright Post-Doctoral Scholar and an URSI Young Scientist Awardee in 2017. 


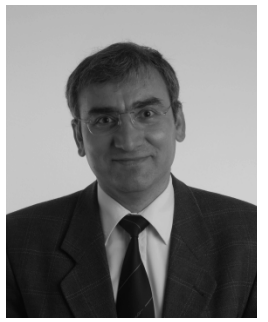

Ayhan Altintas (SM'93) received the B.S. and M.S. degrees from Middle East Technical University (METU), Ankara, Turkey, in 1979 and 1981, respectively, and the Ph.D. degree from The Ohio State University, Columbus, OH, USA, in 1986.

From 1981 to 1987, he was with the ElectroScience Laboratory, The Ohio State University. He is currently a Professor of electrical engineering with Bilkent University, Ankara. He was a Research Fellow and a Guest Professor with Australian National University, Canberra, ACT, Australia, the Tokyo Institute of Technology, Tokyo, Japan, the Technical University of Munich, Munich, Germany, and Concordia University, Montreal, QC, Canada. His current research interests include high-frequency and numerical techniques in electromagnetic scattering and diffraction, propagation modeling and simulation, wireless sensing, and power system monitoring.

Dr. Altintas was the Chairman of the IEEE Turkey Section from 1991 to 1993 and 1995 to 1997. He is a member of Sigma Xi and Phi Kappa Phi. He is currently the President of the URSI Turkish National Committee. He is a Fulbright Scholar, and an Alexander von Humboldt Fellow. He was a recipient of the ElectroScience Laboratory Outstanding Dissertation Award of 1986, the IEEE 1991 Outstanding Student Branch Counselor Award, the 1991 Research Award of the Prof. M. N Parlar Foundation of METU, the Young Scientist Award of Scientific and Technical Research Council of Turkey (Tubitak) in 1996, and the IEEE Third Millennium Medal.



Hilmi Volkan Demir (SM'11) received the B.S. degree in electrical engineering from Bilkent University, Ankara, Turkey, in 1998, and the M.S. and $\mathrm{Ph} . \mathrm{D}$. degrees in electrical engineering from Stanford University, Stanford, CA, USA, in 2000 and 2004, respectively.

$\mathrm{He}$ is a Fellow with the Singapore National Research Foundation and a Full Professor with Nanyang Technological University, Singapore. He is also a Professor of electrical engineering and physics with Bilkent University (his alma mater). He has authored over 250 peer-reviewed research articles in major scientific journals and delivered over 200 invited seminars, lectures, and colloquia. He holds over 30 patents, several of which have currently been used, owned, or licensed by the industry. He has contributed to commercialization and licensing of several new enabling technologies as well as establishing two successful companies. His current research interests include the science of excitonics for high-efficiency light generation and harvesting, nanocrystal optoelectronics, implantable electronics, and wireless sensing.

Dr. Demir was a recipient of the Nanyang Award for Research Excellence, the European Science Foundation European Young Investigator Award, and the Outstanding Young Person in the World Award from the JCI Federation of Young Leaders and Entrepreneurs.



Vakur B. Ertürk (M'00) received the B.S. degree in electrical engineering from Middle East Technical University, Ankara, Turkey, in 1993, and the M.S. and Ph.D. degrees from The Ohio State University, Columbus, OH, USA, in 1996 and 2000, respectively.

$\mathrm{He}$ is currently a Professor with the Electrical and Electronics Engineering Department, Bilkent University, Ankara. His current research interests include the analysis and design of conformal antennas and arrays, wireless sensors for structural health monitoring, numerical techniques, printed circuits, and scattering from and propagation over large terrain profiles.

Dr. Ertürk was the Secretary/Treasurer of the IEEE Turkey Section and the Turkey Chapter of the IEEE Antennas and Propagation, Microwave Theory and Techniques, Electron Devices, and Electromagnetic Compatibility Societies. He was a recipient of the 2005 URSI Young Scientist and the 2007 Turkish Academy of Sciences Distinguished Young Scientist Awards. 